\title{
Segunda citorreducción más quimioterapia hipertérmica intraperitoneal por recaída de seudomixoma peritoneal
}

\author{
Mauricio García ${ }^{1}$, Mario Arturo Abadía², Felipe Solórzano ${ }^{3}$
}

Palabras clave: seudomixoma peritoneal; procedimientos quirúrgicos de citorreducción; quimioterapia del cáncer por perfusión regional; neoplasias del apéndice.

\section{Resumen}

Introducción. El seudomixoma peritoneal es una condición rara caracterizada por la presencia de ascitis mucinosa e implantes peritoneales, en la mayoría de los casos, provenientes de tumores mucinosos del apéndice. El tratamiento primario de esta enfermedad es quirúrgico, y la citorreducción más quimioterapia hipertérmica intraperitoneal es el estándar de tratamiento actual, con supervivencia global a 5 y 10 años hasta de 96 y $68 \%$, respectivamente.

No obstante, es una cirugía con alta morbilidad y considerable mortalidad, que apenas se está introduciendo en Colombia y la experiencia es incipiente. El

1 Médico, cirujano oncólogo, Servicio de Seno y Tejidos Blandos, Instituto Nacional de Cancerología E.S.E., Bogotá, D.C., Colombia

2 Médico, cirujano gastrointestinal, Servicio de Cirugía Gastrointestinal, Instituto Nacional de Cancerología E.S.E., Bogotá, D.C., Colombia

3 Médico, fellow en Cirugía Oncológica, Instituto Nacional de Cancerología E.S.E.-Universidad Militar Nueva Granada, Bogotá, D.C., Colombia.

Fecha de recibido: 22 de septiembre de 2016

Fecha de aprobación: 28 de octubre de 2016

Citar como: García M, Abadía MA, Solórzano F. Segunda citorreducción más quimioterapia hipertérmica intraperitoneal por recaída de seudomixoma peritoneal. Rev Colomb Cir. 2017;32: 193-204. porcentaje de recaida es de 28 a $44 \%$ y existen pocos reportes sobre su manejo; la segunda citorreducción más quimioterapia hipertérmica intraperitoneal parece tener resultados aceptables en cuanto a supervivencia, morbilidad y mortalidad.

Resultados. No hay estudios de segundas intervenciones por recaída peritoneal del seudomixoma en Colombia y, por esta razón, se decidió reportar la experiencia de dos casos del Instituto Nacional de Cancerología, donde, después de una primera citorreducción más quimioterapia hipertérmica intraperitoneal, los pacientes presentaron recaída peritoneal diagnosticada con imágenes durante el seguimiento y fueron sometidos a una nueva cirugía con buen resultado quirúrgico.

Conclusión. La citorreducción secundaria más quimioterapia hipertérmica intraperitoneal es un procedimiento complejo con morbilidad considerable, que debe practicarse en lugares con experiencia y que proporciona al paciente un tratamiento radical $y$, posiblemente, se convierta en el manejo estándar de la recaída.

\section{Introducción}

El seudomixoma peritoneal es una condición rara y enigmática, ahora conocido como un síndrome clínico caracterizado por ascitis mucinosa (material gelatinoso), secundario a la extravasación de moco y una cantidad variable de células tumorales en la superficie peritoneal, de diferente origen, benigno o maligno ${ }^{1,2}$, y caracteri- 
zado por el fenómeno de redistribución ${ }^{3}$. Inicialmente, se pensaba que el origen más común era el ovario; sin embargo, a partir de varios estudios reportados alrededor de los años 90, se identificó al apéndice como el origen en $94 \%$ de los casos de seudomixoma peritoneal ${ }^{4-6}$; otros posibles órganos originarios son el colon y el páncreas ${ }^{7,8}$ $\mathrm{y}$, menos frecuentemente, el uraco ${ }^{9,10}$.

Es una entidad poco común, con una incidencia anual estimada de uno a dos casos por cada millón de personas o de una por cada 1.000 apendicectomías ${ }^{7,11}$.

El tratamiento estándar utilizado actualmente fue descrito por Paul H. Sugarbaker en 1991, como una cirugía de citorreducción más quimioterapia hipertérmica intraperitoneal, abordando esta enfermedad como una local ${ }^{12,13}$. También, diseñó una de las clasificaciones actualmente más utilizadas para graduar la gravedad de la carcinomatosis: el índice de carcinomatosis peritoneal (Peritoneal Cancer Index, PCI) ${ }^{14}$. En otros estudios se ha confirmado la utilidad de esta técnica quirúrgica, con resultados en la supervivencia global a 5 y 10 años entre $52-96 \%$ y $55-68 \%$, respectivamente ${ }^{15-17}$.

Aunque en Estados Unidos y Europa se han reportado grandes series de casos de citorreducción más quimioterapia hipertérmica intraperitoneal ${ }^{15}$, hay pocos reportes sobre la segunda intervención para tratar la recaída tumoral peritoneal por seudomixoma y es una conducta que apenas se está instaurando como estándar. En Latinoamérica, son muy escasas las series de tratamiento primario ${ }^{18-20}$ y nulos los reportes de segunda citorreducción más quimioterapia hipertérmica intraperitoneal para tratar dichas recaídas. En Colombia, este procedimiento es una técnica quirúrgica nueva que se inició en el 2007, según una única serie de 48 casos con diversos tumores primarios, pero sin reporte de experiencia alguna en segundas intervenciones ${ }^{21,22}$.

Es bien sabido que la citorreducción más quimioterapia hipertérmica intraperitoneal es una técnica quirúrgica compleja, que requiere de centros de excelencia y se asocia con altos índices de complicaciones y con reportes de morbilidad del $44 \%$ y mortalidad hasta del 13,8 $\%{ }^{23}$.

En este reporte de dos casos, se quiere presentar la experiencia inicial en el Instituto Nacional de Cancerología E.S.E. en la citorreducción secundaria más quimioterapia hipertérmica intraperitoneal, como reintervención por recaída tumoral por seudomixoma peritoneal, y hacer la revisión bibliográfica sobre la clasificación, el tratamiento actual, los puntos de mayor discusión y los reportes que la avalan.

\section{Caso 1}

Se trata de una mujer de 49 años de edad con antecedentes ginecobstétricos de 5 embarazos, 4 partos y 1 aborto, y sin otros antecedentes personales ni familiares de importancia.

La paciente presentó un cuadro clínico de seis meses de evolución consistente en distensión abdominal progresiva asociada a dolor pélvico irradiado a todo el abdomen.

Consultó inicialmente al Hospital Universitario San Ignacio, donde le practicaron una tomografía computarizada (TC) de abdomen que reveló presencia de ascitis y de una masa quística tabicada de $18 \mathrm{~cm}$ en la cavidad pélvica, sugestiva de tumor de ovario.

Fue sometida a cirugía y se encontró un seudomixoma peritoneal con $3.000 \mathrm{ml}$ de mucina, una masa de 15 $\mathrm{cm}$ en el anexo izquierdo, una de $10 \mathrm{~cm}$ en el anexo derecho, y otra de $5 \mathrm{~cm}$ en apéndice. Se practicó salpingooforectomía bilateral más histerectomía y drenaje de la ascitis mucosa. La revisión de la histopatología en el Instituto Nacional de Cancerología E.S.E., demostró adenomucinosis peritoneal diseminada.

Posteriormente, fue remitida al Instituto Nacional de Cancerología E.S.E., donde refirió nuevamente distensión y dolor abdominal. Se practicó una nueva TC de abdomen en la que se observó ascitis de gran volumen, aumento de la densidad de la grasa intraperitoneal, y colección o masa quística de 53 x $33 \mathrm{~mm}$ en la fosa iliaca izquierda (figura 1). La TC de tórax fue normal y en la colonoscopia se observó una lesión nodular en la válvula ileocecal, de la cual no se tomó biopsia. Se determinó un índice de carcinomatosis peritoneal (PCI) prequirúrgico de $17 \mathrm{y}$ fue sometida a cirugía de citorreducción más quimioterapia hipertérmica intraperitoneal.

En esta primera cirugía (figura 2), se observó nuevamente la presencia de $3.000 \mathrm{ml}$ de mucina con implantes peritoneales en toda la cavidad abdominal (figura 3). Se practicaron: peritonectomía parietal y diafragmática bilaterales (figura 4) y pélvica; hemi- 


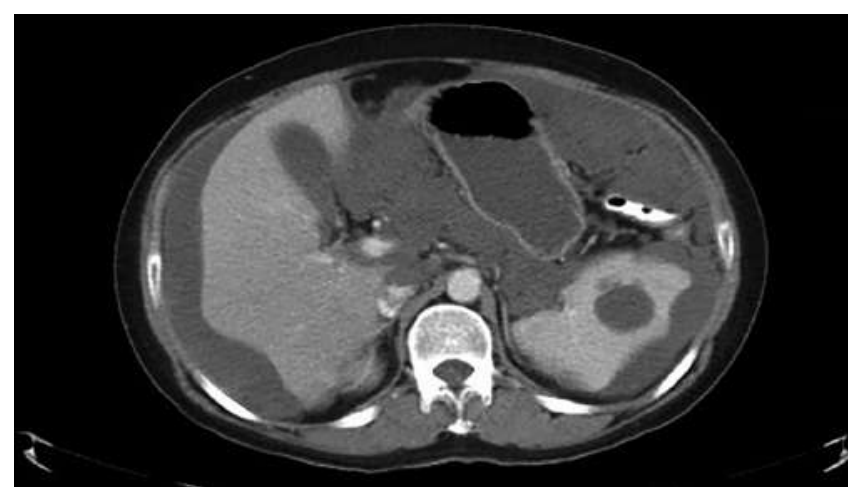

FIgURA 1. TC de abdomen: seudomixoma peritoneal. Se observan áreas hipoecoicas consistentes en mucina en región perihepatica, interasas, periesplénica y hacia pared abdominal anterior izquierda.

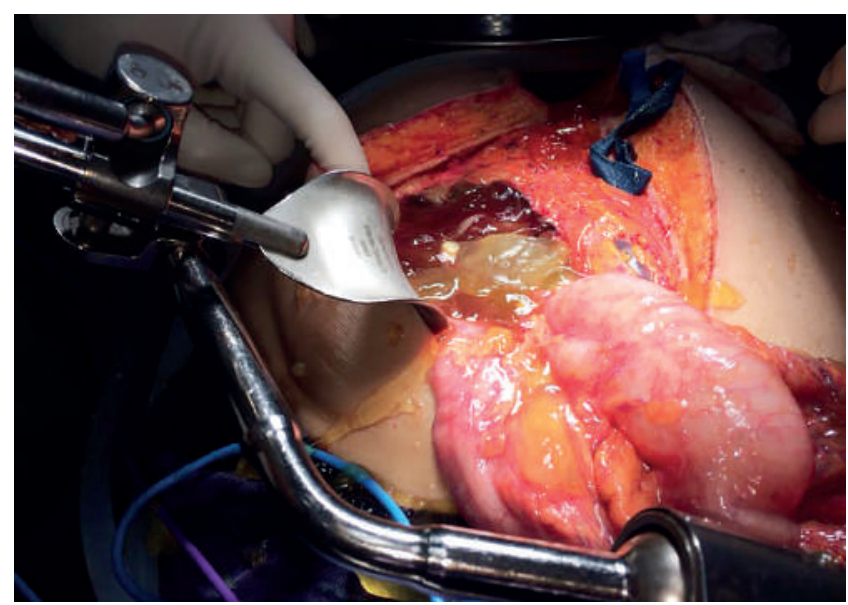

FIgURA 2. Ascitis mucosa. Se evidencia la presencia de mucina libre en la cavidad peritoneal (material parecido a gelatina).

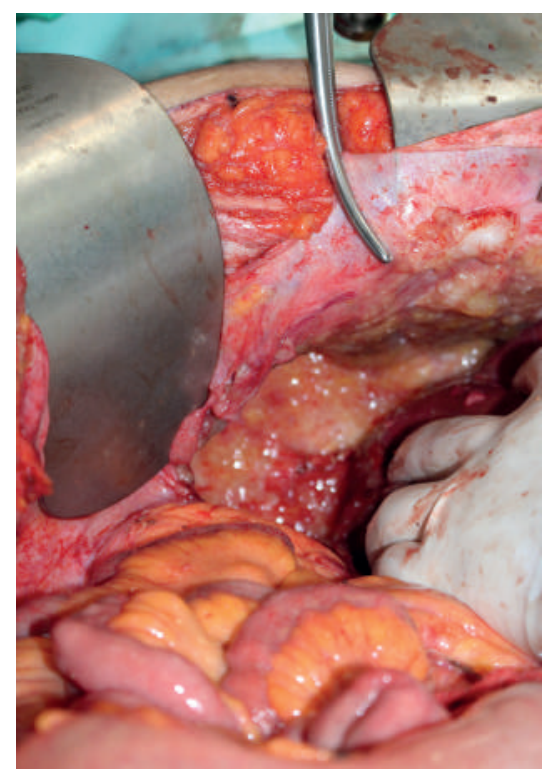

FIgURA 3. Carcinomatosis peritoneal mucosa en la cúpula diafragmática derecha. Se pueden observar los implantes por carcinomatosis mucinosa en el peritoneo diafragmático derecho.

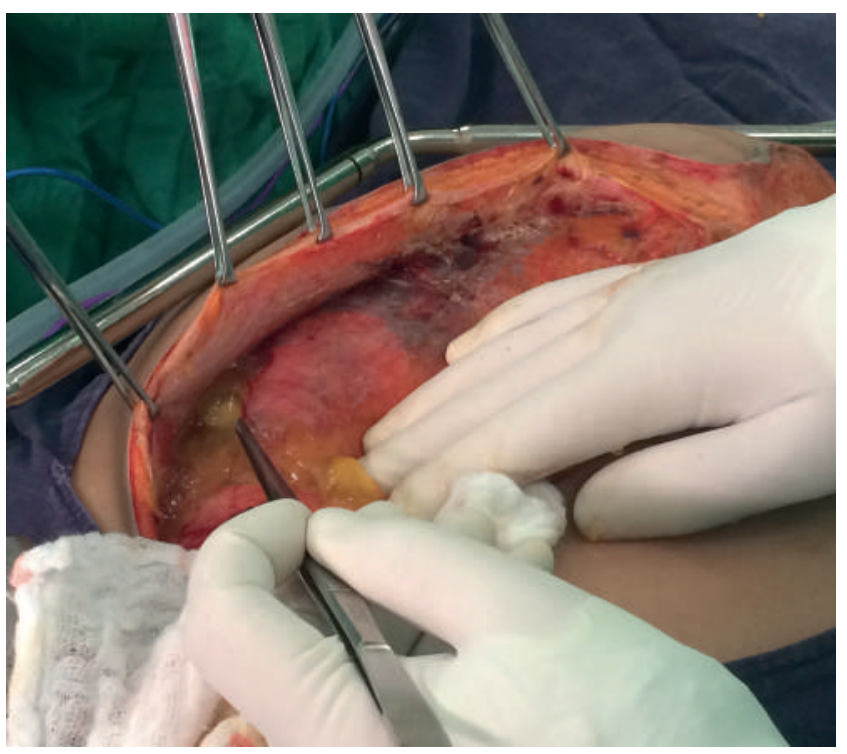

Figura 4. Peritonectomías parietales. Consiste en la resección del peritoneo parietal involucrado por tumor, se habla de peritonectomías parietales las que se hacen en los flancos, sin embargo, también se hacen peritonectomías diafragmáticas y pélvica.

colectomía derecha extendida; resección de la cápsula de Glisson; colecistectomía; omentectomía mayor y menor, ileostomía terminal y toracostomía bilateral, lográndose una citoreducción con resultado óptimo, de grado CC-0 en la escala CCS (Completeness Cytoreductive Score) de Sugarbaker, es decir, sin residuo tumoral macroscópico (figura 5).

Para la quimioterapia intraperitoneal se utilizó carboplatino, $1.300 \mathrm{mg}$ por 90 minutos a $42^{\circ} \mathrm{C}$, mediante la técnica de abdomen cerrado. Durante la resección de la cápsula de Glisson, se presentó sangrado por desgarro en el parénquima del segmento hepático VI, que requirió 'empaquetamiento'. El volumen de sangrado fue de 3.000 $\mathrm{ml}$, aproximadamente, y la duración del procedimiento quirúrgico, de 11 horas y 17 minutos.

La paciente se trasladó a la unidad de cuidados intensivos y recibió asistencia respiratoria mecánica y vasopresores. Al segundo día posoperatorio se sometió a una nueva laparotomía para una segunda revisión, el 'desempaquetamiento' y el cierre de la cavidad abdominal. Sin otra complicación posoperatoria más que un íleo, que solo permitió iniciar la vía oral al noveno día, la paciente permaneció ocho días en la unidad de cuidados intensivos, con cuatro días de asistencia respiratoria mecánica invasiva, y estuvo 10 días más hospitalizada en sala general. 


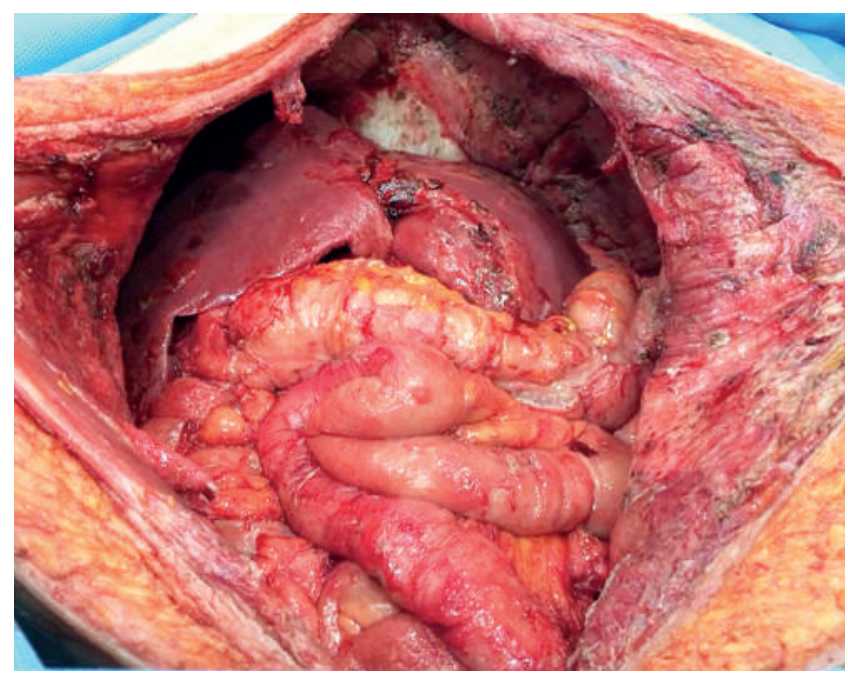

FIgURA 5. Peritonectomías completas y citorreducción de grado CC0. CC-0: cuando no quedan residuos tumorales macroscópicamente. CC-1: cuando quedan lesiones menores de 2,5 mm. CC-2: cuando quedan residuos entre $2,5 \mathrm{~mm}$ y 2,5 cm. CC-3: cuando quedan residuos mayores a $2,5 \mathrm{~cm}$.

El estudio de anatomía patológica demostró adenomucinosis peritoneal diseminada. Durante el seguimiento, en una TC de abdomen tomada cinco meses después de la cirugía, se observó desaparición casi completa de las masas peritoneales quísticas y persistencia de una lesión subdiafragmática de $35 \mathrm{~mm}$; no había ascitis ni lesiones extraperitoneales.

En el séptimo mes posoperatorio, se le practicó cierre de ileostomía por laparotomía. Se encontró un nódulo mucoso sobre el psoas iliaco izquierdo el cual se resecó; la histopatología fue positiva para una neoplasia mucinosa. En un nuevo control a los 16 meses de la cirugía, en el estudio imagenológico se encontró aumento de las lesiones: múltiples lesiones en la cúpula hepática de hasta $10 \mathrm{~mm}$; dos lesiones subcapsulares hepáticas de hasta $19 \mathrm{~mm}$, y otra lesión de 64 x 66 $\mathrm{mm}$, entre el segmento VII y VIII. Todas estas imágenes no tuvieron representación hipermetabólica en la tomografía por emisión de positrones (PET-CT) con 18 FDG. Mediante estos estudios, se calculó un PCI de 3 (figura 6).

A los 20 meses de la primera citorreducción más quimioterapia hipertérmica intraperitoneal, se practicó una segunda intervención por recaída tumoral peritoneal. Se encontró seudomixoma, importantes bridas con el hígado firmemente adherido al diafragma, e implan-

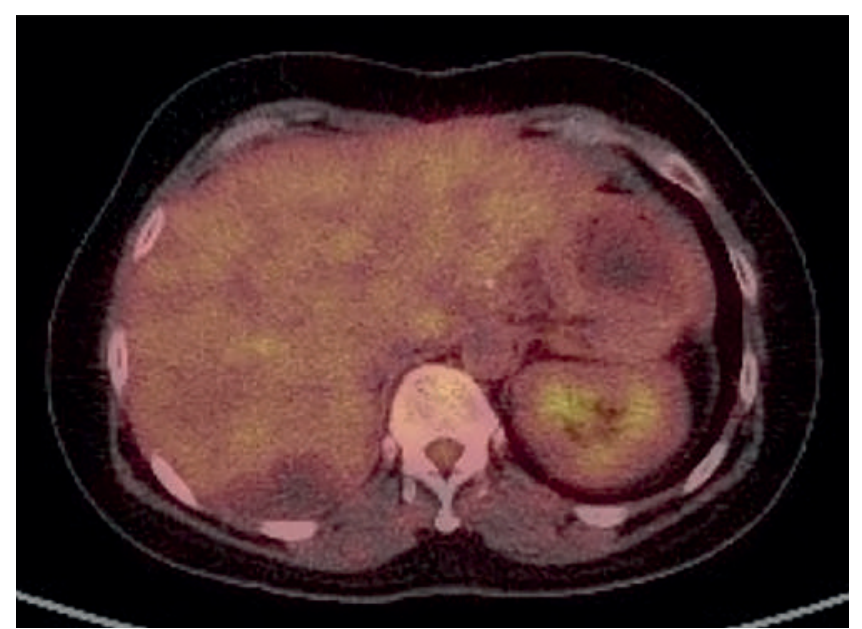

FIGURA 6. PET-CT: recaída peritoneal retrohepática Se observa un área hipoecoica hacia el segmento VI del hígado que no resalta metabólicamente y que corresponde a implante subcapsular de mucina.

tes mucinosos en la cúpula diafragmática derecha, el perigastrio, el hilio hepático, el epiplón menor y el muñón del conducto cístico. Se practicaron: liberación de adherencias, toracostomía derecha por apertura accidental del diafragma, citorreducción completa con resección de implantes peritoneales con resultado óptimo, de grado $\mathrm{CC}-0$, y quimioterapia intraperitoneal con mitomicina, $40 \mathrm{mg}$ por 60 minutos a $42{ }^{\circ} \mathrm{C}$ con técnica cerrada (figura 7). El sangrado intraoperatorio fue de $3.000 \mathrm{ml} \mathrm{y}$, el tiempo quirúrgico, de 7 horas y 30 minutos.

Se hospitalizó en la unidad de cuidados intensivos para vigilancia posoperatoria durante dos días, sin soporte respiratorio ni vasopresor, en cuidados intermedios, cuatro días, y en sala general, seis días, para un total de 12 días de hospitalización. El estudio de histopatología reportó adenomucinosis peritoneal diseminada de bajo grado. La evolución fue buena y en el seguimiento a los tres meses, la TC de abdomen demostró disminución considerable del número de implantes peritoneales con respecto al estudio previo a la cirugía, con lesiones residuales adyacentes a los segmentos hepáticos I, II, III y IV.

En un control a los 14 meses, en la TC se observó desaparición de las lesiones adyacentes al lóbulo hepático derecho, y persistencia de las lesiones adyacentes al segmento IV. Además, el antígeno carcinoembrionario era de $2,3 \mu \mathrm{g} / 1$ y la paciente se encontraba asintomática. 


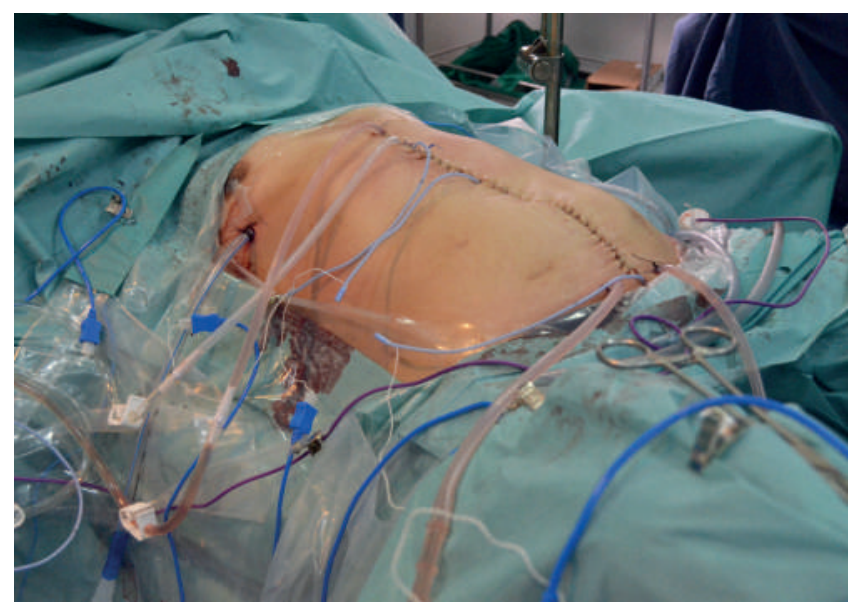

Figura 7. Quimioterapia hipertérmica intraperitoneal, técnica cerrada. Consiste en la aplicación de algún agente de quimioterapia en la cavidad peritoneal en un medio de transporte, como solución de glucosa, a temperatura entre $42-43{ }^{\circ} \mathrm{C}$ por 30 a 90 minutos según la indicación. La técnica cerrada se realiza cerrando la cavidad peritoneal, contrario a la técnica en coliseo que se hace con el abdomen abierto. Cualquiera de las dos técnicas requiere de máquina de perfusión o circulación extracorpórea con calentador.

\section{Caso 2}

Se trata de una mujer de 41 años de edad, con antecedentes de seudomixoma peritoneal por adenocarcinoma de ovario, que había sido sometida a dos cirugías de citorreducción y a una cirugía de citorreducción más quimioterapia hipertérmica intraperitoneal. La primera fue en octubre de 2007 cuando, por sospecha de abdomen agudo, se indicó una laparotomía urgente y se encontró seudomixoma peritoneal con masas bilaterales de los anexos; se practicó salpingooforectomía bilateral, histerectomía y apendicectomía. Inicialmente, la histopatología indicó un adenocarcinoma mucinoso bien diferenciado y se manejó como un tumor primario de ovario. A los seis meses se diagnosticó una recaída mediante imagenología y se manejó de manera expectante.

En mayo de 2010, por presentar distensión y dolor abdominal, se sometió a una citorreducción secundaria del $70 \%$ y, después, recibió tratamiento adyuvante con cisplatino más paclitaxel en cuatro ciclos. Los exámenes de control evidenciaron progresión de la enfermedad peritoneal y fue remitida a uno de los grupos de la ciudad que trabajan en peritoneo.

En la revisión posterior de la histopatología, se informó una neoplasia mucinosa de bajo grado en el apéndice y adenomucinosis peritoneal diseminada.
En mayo de 2011, se practicó una citorreducción más quimioterapia hipertérmica intraperitoneal con mitomicina más doxorrubicina venosa con 5 fluorouracilo intravenoso (bidireccional), más quimioterapia intraperitoneal posoperatoria temprana (Early Postoperative Intraperitoneal Chemotherapy, EPIC) con 5 florouracilo durante cuatro días. En la anatomía patológica se observó adenomucinosis peritoneal diseminada. A los 18 meses del posoperatorio, se presentó una recaída con demostración, mediante TC abdominal, de una lesión nodular retrovesical y otra pararrectal izquierda.

La paciente permaneció asintomática, pero en el examen físico se detectó un nódulo en la pared abdominal, por lo cual fue remitida al Servicio de Cirugía Gastrointestinal del Instituto Nacional de Cancerología. En una nueva evaluación con imágenes, no se evidenció enfermedad extraperitoneal pero sí progresión abdominal (figura 8).

Por decisión de la junta quirúrgica, se sometió a una segunda citorreducción más quimioterapia hipertérmica intraperitoneal a los cuatro años y medio después de la primera intervención de citorreducción. En esta intervención, el índice de carcinomatosis peritoneal (PCI) intraoperatorio fue de 16 y se encontró: importante cantidad de adherencias; múltiples implantes en la cicatriz de la laparotomía anterior, un gran tumor pélvico mucinoso de $20 \times 20 \mathrm{~cm}$ que se extendía hasta la fosa iliaca izquierda y, también, implantes en el ligamento gastrohepático con compromiso del hiato de Winslow;

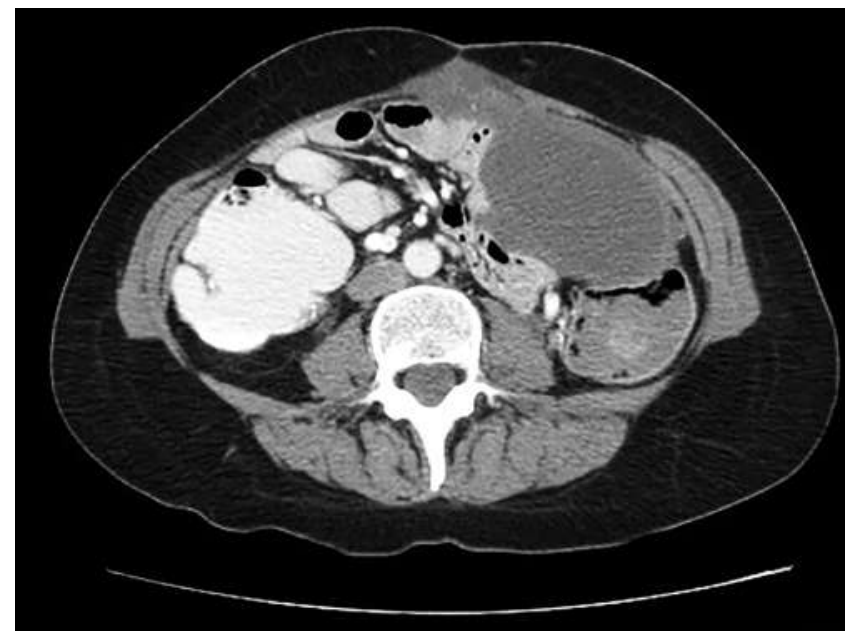

Figura 8. Recaida intraperitoneal de seudomixoma. Se observa lesión hipoecoica intraperitoneal hacia flanco izquierdo y que contacta la pared abdominal anterior y estructuras intestinales. 
no se evidenció recaída en las anastomosis ileocólicas antiguas.

Se procedió a liberar las adherencias; se realizaron peritonectomías del neoperitoneo en las cúpulas diafragmáticas, las goteras parietocólicas y la pelvis; se resecaron la masa pélvica mucosa y los implantes en la vena iliaca izquierda, en placa hiliar hepática, el hilio hepático y en el domo vesical; se administró la quimioterapia hipertérmica intraperiteonal con $15 \mathrm{mg}$ de mitomicina más $20 \mathrm{mg}$ de doxorrubicina, mediante técnica cerrada a $41{ }^{\circ} \mathrm{C}$ durante 60 minutos; finalmente, se cerró la pared abdominal y se dejaron drenajes en la cavidad abdominal, y no se dejaron sondas en tórax. Hubo un sangrado de $1.300 \mathrm{ml}$, aproximadamente, y la duración del procedimiento fue de 10 horas.

La paciente permaneció cuatro días con soporte respiratorio y vasopresor con norepinefrina en la unidad de cuidados intensivos, sin presentar complicaciones posoperatorias. La vía oral solo se pudo reiniciar en el octavo día posoperatorio, por presentar un íleo prolongado. Se dio de alta hospitalaria el día 23 después de la cirugía.

En el estudio de histopatología se reportó compromiso por neoplasia mucosa bien diferenciada (adenomucinosis peritoneal diseminada).

En el seguimiento posoperatorio a los tres meses, permanecía sin complicaciones y sin signos de recidiva tumoral. En el control a los nueve meses, la TC no demostró recaída peritoneal y no había complicaciones clínicas.

\section{Discusión}

En 1842, Karl Rokitansky diagnosticó un mucocele del apéndice; sin embargo, el primer reporte de seudomixoma peritoneal fue hecho por Werth en 1884, quien lo atribuyó a una neoplasia del ovario. Frankel, en 1901, describió un seudomixoma peritoneal asociado a un quiste del apéndice ${ }^{24}$.

Aunque el diagnóstico de seudomixoma se hace mediante los hallazgos macroscópicos de ascitis mucinosa (material parecido a la gelatina en la cavidad peritoneal), la determinación de la etiología no ha sido tan fácil y ha requerido estudios de biología molecular para aclarar que, en la mayoría de los casos, este tipo de carcinomatosis proviene del apéndice y no del ovario ${ }^{25-28}$, confusión favorecida porque la prevalencia en mujeres es dos a tres veces mayor que en hombres ${ }^{29}$.

En los dos casos presentados, el origen del seudomixoma se atribuyó inicialmente al ovario y fue manejado como tal; generalmente, su etiología se aclara en los centros especializados en enfermedad peritoneal. Por otra parte, el cuadro clínico inicial de abdomen agudo que simula una apendicitis y la distensión abdominal progresiva con masas ováricas evidentes por imagenología, son las dos presentaciones más comunes del seudomixoma peritoneal, algo ya descrito por Esquivel en el $2000^{30}$.

Entre los tumores del apéndice, históricamente se ha reconocido al carcinoide (neuroendocrino) como el más común, correspondiendo a más de $50 \%$ de los casos ${ }^{31}$. No obstante, en el Surveillance, Epidemiology and End Results (SEER) del National Cancer Institute de los Estados Unidos, se reportó en el 2007 una mayor incidencia de tumores epiteliales no neuroendocrinos, también llamados "tumores epiteliales"; de estos, el adenocarcinoma mucinoso es el tipo histológico más frecuente $(37 \%)$, seguido del adenocarcinoma de tipo intestinal $(27 \%)$, el tumor carcinoide de células caliciformes $(18,9 \%)$, los tumores carcinoides malignos (11\%) y, por último, los tumores de células en anillo de sello $(5,5 \%)^{32}$. La incidencia global de los tumores de apéndice es baja; se estima que, de todas las apendicectomías, se encuentran lesiones tumorales en 0,67 a $0,9 \%$ y, neoplasias malignas, en 0,1 a $0,52 \%$ ${ }^{31,33}$. En un estudio de población holandesa del 2008, se informó que el $10 \%$ de los pacientes con lesiones epiteliales del apéndice desarrollan seudomixoma peritoneal, lo cual puede aumentar a $20 \%$ si se trata de un adenocarcinoma, lo que ha llevado a estimar una incidencia de seudomixoma de 1 por cada 1.000 apendicectomías ${ }^{11}$.

El amplio espectro de lesiones del apéndice (que incluye lesiones benignas, como los adenomas y los tumores mucinosos, o las malignas parecidas al cáncer de colon), y los hallazgos en el peritoneo cuando existe diseminación de moco con o sin células de diferentes grados histológicos, han generado múltiples interpretaciones de diferentes autores, al intentar clasificar los hallazgos microscópicos, tanto del apéndice como del peritoneo ${ }^{34-39}$. 
Esta situación ha generado más confusión y diferencias entre los autores, a la hora de estudiar y reportar la biología tumoral. Una de las clasificaciones de los hallazgos histológicos en el peritoneo más usadas, es la de Ronnett, et al., quienes propusieron el nombre de adenomucinosis peritoneal diseminada (Disseminated Peritoneal Adenomucinosis, DPAM), para los cambios epiteliales de bajo grado, y el de carcinomatosis mucinosa peritoneal (Peritoneal Mucinous Carcinomatosis, PMCA), para los de alto grado ${ }^{34}$. Aunque también se describió una lesión discordante como carcinomatosis mucinosa peritoneal intermedia (Intermediate Peritoneal Mucinous Carcinomatosis, PMCA-I), este nombre no se popularizó pues sus hallazgos generan confusión, ya que el pronóstico se asemeja a los de la carcinomatosis mucinosa peritoneal, en unos artículos, y a los de la adenomucinosis peritoneal diseminada, en otros ${ }^{37,40}$

En un intento por homologar la terminología y los reportes de los diferentes grupos alrededor del mundo, a principios de 2016 se publicó el primer consenso sobre la clasificación y el reporte anatomo-patológico del seudomixoma peritoneal, en el cual participaron 71 expertos mundiales en peritoneo, incluidos patólogos, cirujanos oncólogos y oncólogos clínicos. En este consenso se adoptó una nueva terminología según los hallazgos histológicos, así: mucina acelular (mucina sin células epiteliales); carcinomatosis peritoneal de bajo grado o DPAM (seudomixoma con características histológicos de bajo grado); carcinomatosis peritoneal de alto grado o PMCA (seudomixoma con características histológicas de alto grado), y carcinomatosis peritoneal de alto grado con células en anillo de sello o PMCA-S (seudomixoma con características de alto grado con células en anillo de sello).

También, se llegó a un consenso sobre la terminología de los hallazgos histológicos apendiculares, la cual se debe seguir usando. Se estableció, también, que para el estadio IV del $7 \mathrm{mo}$ TNM, se debe tener en cuenta la histología del peritoneo y no la del apéndice. Además, se reforzó el concepto del seudomixoma peritoneal como un síndrome clínico y como una entidad que se debe considerar maligna ${ }^{41}$.

En una revisión sistemática sobre el tratamiento primario del seudomixoma peritoneal con citorreducción más quimioterapia hipertérmica intraperitoneal, Bryant, et al., reportaron supervivencia global a cinco años de 70 a $86 \%$ y, a 10 años, una de 60 a $68 \%{ }^{17}$, y Yan, et al., en otra revisión sistemática, reportaron una supervivencia global a cinco años de 52 a $92 \%$ y, a 10 años, una de $55 \%{ }^{15}$.

Existen muchos otros estudios con resultados similares e, incluso, discriminan la supervivencia global según los diferentes factores pronóstico, como la histopatología peritoneal, el PCI y la escala CCS; entre estos, los de Ronnett, et al., quienes calcularon una supervivencia global para la adenomucinosis peritoneal diseminada a 5 y 10 años de $75 \%$ y $68 \%$, y para la carcinomatosis mucosa peritoneal, de $14 \%$ y $3 \%$, respectivamente ${ }^{34,40}$. Jiménez $\mathrm{W}$, et al., reportaron una supervivencia global a cinco años de $83 \%$ versus $41 \%$ para DPAM y PMCA respectivamente. Estas cifras varían según el PCI y con los grados CC 0 -1, con supervivencia global a cinco años de $88 \%$ en casos de DPAM con CC $0-1$ y de $48 \%$ con los grados CC 2-3 ( $\mathrm{p}=0,021$ ); en PMCA con grados CC $0-1$ fue de $44 \%$ y, con grados CC 2-3, de $0 \%$. Al comparar el PCI, la supervivencia global a cinco años en casos de DPAM con un PCI menor de 20 fue de $100 \%$ $y$, con un PCI mayor de 20, fue de $80 \%(p=0,145)$; en la PMCA con un PCI menor de 20 fue de $60 \% \mathrm{y}$, con un PCI mayor de 20 , de $36 \%(\mathrm{p}=0,002)^{42}$. La citorreduccion más quimioterapia hipertérmica intraperitoneal es una cirugía agresiva con altos porcentajes de complicaciones, con morbilidad entre 33 y $56 \%$, y mortalidad entre 0 y $18 \%{ }^{15}$; sin embargo, autores con amplia experiencia y buen número de pacientes, como Sugarbaker, reportan $2 \%$ de mortalidad intrahospitalaria o a 30 días, y $19 \%$ de complicaciones de grado 3 y $4{ }^{43}$.

En el Fifth International Workshop on Peritoneal Surface Maligancy (Milán, Italia, 2006), se acordó unánimemente que la citorreducción más la quimioterapia hipertérmica intraperitoneal era el tratamiento estándar para los tumores mucinosos asociados a diseminación peritoneal ${ }^{44}$, con base en múltiples estudios con resultados favorables en supervivencia, morbilidad y mortalidad ${ }^{15,45-50}$. Sin embargo, no existe un consenso sobre el manejo de la recaída peritoneal del seudomixoma. Antes de los años noventa, la citorreducción quirúrgica sin quimioterapia intraperitoneal (debulking) era el tratamiento inicial para las recurrencias peritoneales, lográndose supervivencia global a 10 años de 26 a $60 \%$, y recurrencia tumoral de 76 a $91 \%{ }^{29,51,52}$. Eventualmente, estos pacientes tratados con citorreducción (debulking) a repetición 
fallecen por obstrucción intestinal, inanición terminal o complicaciones quirúrgicas.

Hasta ahora, son pocos los autores que han abordado este tema de las recurrencias en la era de la citorreducción más quimioterapia hipertérmica intraperitoneal, y se han reportado pocas series de casos donde se evalúe este esquema terapéutico en un segundo o un tercer intento de controlar la enfermedad ${ }^{53-55}$.

Algunos autores han reportado recurrencias hasta de $28 \%$ de los casos después de citorreducción completa más quimioterapia hipertérmica intraperitoneal ${ }^{56,57}$; sin embargo, en un estudio de Smeenk, et al., se reportó 44 $\%$ de recurrencia después de este tratamiento, con una supervivencia libre de enfermedad de $43 \%$ a tres años y de $37 \%$ a cinco años, y una mediana de intervalo libre de enfermedad de 25 meses ${ }^{58}$.

Chua, et al., en una serie de 113 pacientes con seudomixoma peritoneal de bajo grado (DPAM o PMCA-I), reportaron recurrencia en 41 (36 \%) casos; lo que llama la atención y no se había reportado en otros artículos, es que en cinco (12\%) de esos pacientes con recurrencia se comprometían el tórax y el abdomen. En el análisis multivariado incluyeron algunos factores asociados a la recurrencia temprana (primeros 12 meses), como: sexo masculino, elevación de marcadores tumorales como el CA-125, PCI mayor de 25, transfusión de más de 6 unidades de glóbulos rojos, grado de citorreducción CC-2 y transfusión de más de 10 unidades de plasma fresco congelado ${ }^{59}$. Algunos de estos factores, como la extensión de la cirugía previa con disección de más de cinco regiones, PCI mayor de 20 e histopatología indicativa de PMCA-I o PMCA, ya habían sido expuestos por Yan, et al., y mostraron significancia estadística como factores de riesgo para recurrencia; por el contrario, el sexo, la edad menor de 50 años, los ganglios positivos, y un grado CC-1, no alcanzaron significancia estadística para supervivencia libre de enfermedad ${ }^{56}$.

Esquivel, et al. reportaron una serie de 98 pacientes con una segunda cirugía por recidiva de seudomixoma peritoneal, con una supervivencia global a cinco años de 73,6 \%. En el análisis de subgrupos se observó que el mejor pronóstico de supervivencia global se presentaba cuando, en la segunda citorreducción, el PCI era menos del $50 \%$ del inicial $(\mathrm{p}<0,0001)$; cuando la citorreducción es completa (CC 0-1) en la segunda intervención, la supervivencia a cinco años es de $85 \%$, en comparación con el $44 \%$ cuando es incompleta $(\mathrm{p}=0,001)$. No hubo diferencia estadísticamente significativa según la indicación de la segunda cirugía, excepto en casos de obstrucción intestinal, en cuyo caso el pronóstico es reservado y la supervivencia a cinco años disminuye a $28 \%{ }^{53}$.

Smeenk, et al., reportaron una serie de 11 pacientes sometidos a un segundo procedimiento de citorreducción más quimioterapia hipertérmica intraperitoneal, con una probabilidad calculada de supervivencia relacionada con la enfermedad del 77,8 \% a tres años y una probabilidad de supervivencia libre de enfermedad de $33,3 \%{ }^{58}$.

Brouquet, et al., reportaron una serie de 20 pacientes con reintervención por recidiva tumoral: 12 con seudomixoma peritoneal, cuatro con cáncer de colon, tres con mesotelioma y uno con un tumor carcinoide; no informaron la supervivencia global ni la libre de enfermedad discriminada para seudomixoma, pero sí una mortalidad perioperatoria de $4 \%$ y una morbilidad asociada al procedimiento de $48,1 \%{ }^{60}$.

Otras dos series de casos con una segunda cirugía de citorreducción más quimioterapia hipertérmica intraperitoneal, por recaída de varios tipos de tumor primario (ovario, colorrectal, seudomixoma, mesotelioma, cáncer gástrico), confirmaron complicaciones en 43,5 a $73 \%$ de los pacientes, que son más altas que las presentadas en la primera citorreducción. Ambos artículos concuerdan en que un grado $\mathrm{CC}-0$ o CC-1 en la citorreducción es factor predictivo de mejor supervivencia y solo en uno de ellos se reporta una segunda recaída en $63 \%$ de los casos después de segunda intervención ${ }^{55,61}$.

Sardi, et al., reportaron una serie de 26 pacientes con repetición de la citorreducción por recaída de seudomixoma peritoneal, cuatro de los cuales requirieron tres cirugías de citorreducción. La supervivencia global para la primera cirugía fue de $100 \%, 82,7 \%, 53,6 \%$ y $45,9 \%$, a uno, tres, cinco y 10 años, respectivamente. La supervivencia global para la segunda cirugía fue de $90,9 \%, 54,3 \%$ y $33,9 \%$, a uno, tres y cinco años, respectivamente. Sin embargo, cuando discriminan para DPAM, la supervivencia a cinco años después de la primera cirugía es de $90 \% \mathrm{y}$, de la segunda cirugía, de $100 \%$; mientras que, para PMCA, la supervivencia a cinco años fue de $32 \%$ después de la primera cirugía $\mathrm{y}$, después de la segunda, de $0 \%{ }^{54}$. 


\section{Conclusión}

Se presentan dos casos de reintervención por recaída peritoneal de seudomixoma, tratados exitosamente en el Instituto Nacional de Cancerología con una segunda cirugía de citorreducción más quimioterapia hipertérmica intraperitoneal, como la experiencia inicial en esta institución y en Colombia.

Por tratarse de los dos primeros casos, no es posible el análisis estadístico de los resultados ni sacar conclusiones sólidas. Según la revisión bibliográfica, este tratamiento parece ser una opción viable para pacientes con recaída peritoneal, con adecuados resultados en cuanto a la supervivencia global.

Estos buenos resultados se obtienen si la escogencia de los candidatos para la reintervención es adecuada. Se debe tener en cuenta que una segunda o tercera cirugía citorreductora puede tener más complicaciones perio- peratorias que la primera cirugía; por lo tanto, como no existen guías o criterios claros para escoger los mejores candidatos, depende del buen criterio y la experiencia del cirujano lograr la mejor supervivencia con el menor riesgo operatorio posible.

Tratándose de un procedimiento que apenas empieza a practicarse en Colombia, queremos hacer un llamado a conformar grupos multidisciplinarios (cirujanos supraespecialistas, oncólogos clínicos, anestesiólogos, patólogos, intensivistas, psicólogos, rehabilitadores físicos, etc.) que observen sus indicaciones o límites en casos de seudomixoma y de otros tumores primarios, pues es la única forma para un resultado exitoso. Es una cirugía con gran morbilidad y mortalidad, de alto costo y sacrificio para el personal de la salud; por lo tanto, se requiere lograr una mejor supervivencia para que tenga validez, se justifique el esfuerzo y pueda persistir en el tiempo este tipo de cirugía en nuestro medio.

\title{
Second cytoreduction plus intraperitoneal hyperthermic chemotherapy in recurrence of pseudomyxoma peritonei
}

\begin{abstract}
Introduction: Peritoneal pseudomyxoma is a rare condition characterized by mucinous ascitis and peritoneal implants, originating from mucinous tumors of the apendix in the majority of cases. Primary treatment is surgical resection, with cytoreduction surgery plus hiperthermic intraperitoneal chemotherapy as the current standard of care, with $96 \%$ and $68 \% 5$ and 10 year overall survival rates.

Nonetheless, it is a surgical procedure associated with high morbidity and considerable mortality, with initial experience in Colombia. Recurrence is estimated between $28 \%$ and $44 \%$ and few reports address the management of recurrence peritoneal pseudomyxoma; second cytoreduction surgery plus hiperthermic intraperitoneal chemotherapy $(C R S+H I P E C)$ seems to have acceptable results in terms of survival, morbidity and mortality. No studies of second CRS + HIPEC have been reported in Colombia; this is why we decided to publish two cases treated at the Instituto Nacional de Cancerología, Bogotá, Colombia, who had recurrence after a fist CRS + HIPEC, diagnosed by follow up images and who were taken to a second surgical treatment with good results.

Conclusion: Second CRS + HIPEC is a technically challenging procedure with considerable morbidity that should only be performed in specialized and experienced centers, a radical form of treatment that could possibly become the standard of choice for recurrence.
\end{abstract}

Key words: Pseudomyxoma peritonei; cytoreduction surgical procedures; chemotherapy, cancer, regional perfusion; appendiceal neoplasms. 


\section{Referencias}

1. Limber GK, King RE, Silverberg SG. Pseudomyxoma peritonaei: A report of ten cases. Ann Surg. 1973;178:587-93.

2. Sugarbaker PH, Ronnett BM, Archer A, Averbach AM, Bland R, Chang D, et al. Pseudomyxoma peritonei syndrome. Adv Surg. 1996;30:233-80.

3. Sugarbaker PH. Pseudomyxoma peritonei. A cancer whose biology is characterized by a redistribution phenomenon. Ann Surg. 1994;219:109-11.

4. Prayson RA, Hart WR, Petras RE. Pseudomyxoma peritonei. A clinicopathologic study of 19 cases with emphasis on site of origin and nature of associated ovarian tumors. Am J Surg Pathol. 1994;18:591-603.

5. Seidman JD, Elsayed AM, Sobin LH, Tavassoli FA. Association of mucinous tumors of the ovary and appendix. A clinicopathologic study of 25 cases. Am J Surg Pathol. 1993;17:22-34.

6. Young RH, Gilks CB, Scully RE. Mucinous tumors of the appendix associated with mucinous tumors of the ovary and pseudomyxoma peritonei. A clinico-pathological analysis of 22 cases supporting an origin in the appendix. Am J Surg Pathol. 1991;15:415-29.

7. Mukherjee A, Parvaiz A, Cecil TD, Moran BJ. Pseudomyxoma peritonei usually originates from the appendix: A review of the evidence. Eur J Gynaecol Oncol. 2004;25:411-4.

8. Rosenberger LH, Stein LH, Witkiewicz AK, Kennedy EP, Yeo CJ. Intraductal papillary mucinous neoplasm (IPMN) with extrapancreatic mucin: A case series and review of the literature. $\mathrm{J}$ Gastrointest Surg. 2012;16:762-70.

9. Agrawal AK, Bobinski P, Grzebieniak Z, Rudnicki J, Marek G, Kobielak P, et al. Pseudomyxoma peritonei originating from urachus-case report and review of the literature. Curr Oncol. 2014;21:e155-65.

10. Soto-Delgado M, Pedrero-Márquez G, Varo-Solís C, RodríguezRubio Cortadellas FO, Sánchez-Bernal C, González-Moreno D. Adenocarcinoma mucinoso de uraco y pseudomixoma peritoneal. Actas Urológicas Españolas. 2006;30:222-6.

11. Smeenk RM, van Velthuysen MLF, Verwaal VJ, Zoetmulder FA. Appendiceal neoplasms and pseudomyxoma peritonei: A population based study. Eur J Surg Oncol. 2008;34:196-201.

12. Sugarbaker PH. Cytoreductive surgery and intraperitoneal chemotherapy with peritoneal spread of cystadenocarcinoma. Eur J Surg Suppl. 1991;561:75-82.

13. Sugarbaker PH. Peritonectomy procedures. Surg Oncol Clin N Am. 2003;12:703-2.

14. Gilly FN, Cotte E, Brigand C, Monneuse O, Beaujard AC, Freyer $\mathrm{G}$, et al. Quantitative prognostic indices in peritoneal carcinomatosis. Eur J Surg Oncol. 2006;32:597-601.

15. Yan TD, Black D, Savady R, Sugarbaker PH. A systematic review on the efficacy of cytoreductive surgery and perioperative intraperitoneal chemotherapy for pseudomyxoma peritonei. Ann Surg Oncol. 2007;14:484-92.
16. González-Moreno S, Sugarbaker PH. Right hemicolectomy does not confer a survival advantage in patients with mucinous carcinoma of the appendix and peritoneal seeding. Br J Surg. 2004;91:304-11.

17. Bryant J, Clegg AJ, Sidhu MK, Brodin H, Royle P, Davidson P. Systematic review of the Sugarbaker procedure for pseudomyxoma peritonei. Br J Surg. 2005;92:153-8.

18. Rabelo FEF, de Queiroz FL, do Rego RSN, da Costa BXM, Lamounier PC, de Oliveira TAN, et al. Multimodal treatment of peritoneal malignancies - results of the implantation in a tertiary hospital. Journal of Coloproctology (Rio de Janeiro). 2012;32:106-12.

19. López-Basave HN, Morales-Vásquez F, Ruiz-Molina JM, González-Enciso A, Namendys-Silva SA, Medina-Castro JM, et al. Morbidity and mortality of cytoreductive surgery with hyperthermic intraperitoneal chemotherapy: National Cancer Institute, Mexico City, Mexico. ISRN Oncol. 2011;2011:526384.

20. García-Matus R, Hernández-Hernández CA, Leyva-García O, Vásquez-Ciriaco S, Flores-Ayala G, Navarro-Hernández Q, et al. Cytoreductive surgery and hyperthermic intraperitoneal chemotherapy in the treatment of peritoneal carcinomatosis: Initial experience in Oaxaca, México. Am Surg. 2012;78:942-6.

21. Otero J, Árias F, Londoño E, Mora M, Becerra H, Cortés N, et al. Peritonectomía radical y quimioterapia intraperitoneal: cinco años de experiencia en la Fundación Santa Fe de Bogotá (ONCOL Group - Estudio ATIA). Rev Colomb Hematol Oncol. 2013;2:22-33.

22. Árias F, Otero JM, Guerrero C, Cardona AF, Vargas CA, Carranza $\mathrm{H}$, et al. Pseudomyxoma peritonei: First cases treated by radical cytoreductive surgery and hyperthermic intraperitoneal chemotherapy. Rev Colomb Cir. 2009;24:184-94.

23. Elias D, Laurent S, Antoun S, Duvillard P, Ducreux M, Pocard M, et al. Pseudomyxoma peritonei treated with complete resection and immediate intraperitoneal chemotherapy. Gastroenterol Clin Biol. 2003;27:407-12.

24. Smeenk RM, Bruin SC, van Velthuysen M-LF, Verwaal VJ. Pseudomyxoma peritonei. Curr Probl Surg. 2008;45:527-75.

25. Cuatrecasas M, Matias-Guiu X, Prat J. Synchronous mucinous tumors of the appendix and the ovary associated with pseudomyxoma peritonei. A clinicopathologic study of six cases with comparative analysis of c-Ki-ras mutations. Am J Surg Pathol. 1996;20:739-46.

26. Chuaqui RF, Zhuang Z, Emmert-Buck MR, Bryant BR, Nogales F, Tavassoli FA, et al. Genetic analysis of synchronous mucinous tumors of the ovary and appendix. Hum Pathol. 1996;27:165-71.

27. Young RH. From Krukenberg to today: The ever present problems posed by metastatic tumors in the ovary: Part I. Historical perspective, general principles, mucinous tumors including the Krukenberg tumor. Adv Anat Pathol. 2006;13:205-27.

28. Young RH. Pseudomyxoma peritonei and selected other aspects of the spread of appendiceal neoplasms. Semin Diagn Pathol. 2004;21:134-50. 
29. Smith JW, Kemeny N, Caldwell C, Banner P, Sigurdson E, Huvos A. Pseudomyxoma peritonei of appendiceal origin. The Memorial Sloan-Kettering Cancer Center experience. Cancer. 1992;70:396-401.

30. Esquivel J, Sugarbaker PH. Clinical presentation of the pseudomyxoma peritonei syndrome. Br J Surg. 2000;87:1414-8.

31. Connor SJ, Hanna GB, Frizelle FA. Appendiceal tumors: Retrospective clinicopathologic analysis of appendiceal tumors from 7,970 appendectomies. Dis Colon Rectum. 1998;41:75-80.

32. Turaga KK, Pappas SG, Gamblin TC. Importance of histologic subtype in the staging of appendiceal tumors. Ann Surg Oncol. 2012;19:1379-85.

33. Marudanayagam R, Williams GT, Rees BI. Review of the pathological results of 2660 appendicectomy specimens. J Gastroenterol. 2006;41:745-9.

34. Ronnett BM, Zahn CM, Kurman RJ, Kass ME, Sugarbaker $\mathrm{PH}$, Shmookler BM. Disseminated peritoneal adenomucinosis and peritoneal mucinous carcinomatosis. A clinicopathologic analysis of 109 cases with emphasis on distinguishing pathologic features, site of origin, prognosis, and relationship to "pseudomyxoma peritonei.” Am J Surg Pathol. 1995;19:1390-408.

35. Misdraji J, Yantiss RK, Graeme-Cook FM, Balis UJ, Young RH. Appendiceal mucinous neoplasms: A clinico-pathologic analysis of 107 cases. Am J Surg Pathol. 2003;27:1089-103.

36. Pai RK, Beck AH, Norton JA, Longacre TA. Appendiceal mucinous neoplasms: Clinicopathologic study of 116 cases with analysis of factors predicting recurrence. Am J Surg Pathol. 2009;33:1425-39.

37. Bradley RF, Stewart JH, Russell GB, Levine EA, Geisinger KR. Pseudomyxoma peritonei of appendiceal origin: A clinico-pathologic analysis of 101 patients uniformly treated at a single institution, with literature review. Am J Surg Pathol. 2006;30:551-9.

38. Bosman FT, Carneiro F, Hruban RH, Theise ND. WHO classification of tumours of the digestive system. Fourth edition. Lyons: IARC; 2010. p. 417.

39. Carr NJ, McCarthy WF, Sobin LH. Epithelial noncarcinoid tumors and tumor-like lesions of the appendix. A clinicopathologic study of 184 patients with a multivariate analysis of prognostic factors. Cancer. 1995;75:757-68.

40. Ronnett BM, Yan H, Kurman RJ, Shmookler BM, Wu L, Sugarbaker PH. Patients with pseudomyxoma peritonei associated with disseminated peritoneal adenomucinosis have a significantly more favorable prognosis than patients with peritoneal mucinous carcinomatosis. Cancer. 2001;92:85-91.

41. Carr NJ, Cecil TD, Mohamed F, Sobin LH, Sugarbaker PH, González-Moreno S, et al. A consensus for classification and pathologic reporting of pseudomyxoma peritonei and associated appendiceal neoplasia: The results of the Peritoneal Surface Oncology Group International (PSOGI) Modified Delphi Process. Am J Surg Pathol. 2016;40:14-26.

42. Jiménez W, Sardi A, Nieroda C, Sittig M, Milovanov V, Núñez $\mathrm{M}$, et al. Predictive and prognostic survival factors in peritoneal carcinomatosis from appendiceal cancer after cytoreductive surgery with hyperthermic intraperitoneal chemotherapy. Ann Surg Oncol. 2014;21:4218-25.

43. Sugarbaker PH, Alderman R, Edwards G, Marquardt CE, Gushchin V, Esquivel J, et al. Prospective morbidity and mortality assessment of cytoreductive surgery plus perioperative intraperitoneal chemotherapy to treat peritoneal dissemination of appendiceal mucinous malignancy. Ann Surg Oncol. 2006;13:635-44

44. Moran B, Baratti D, Yan TD, Kusamura S, Deraco M. Consensus statement on the loco-regional treatment of appendiceal mucinous neoplasms with peritoneal dissemination (pseudomyxoma peritonei). J Surg Oncol. 2008;98:277-82.

45. Moran BJ, Cecil TD. The etiology, clinical presentation, and management of pseudomyxoma peritonei. Surg Oncol Clin N Am. 2003;12:585-603.

46. Sugarbaker PH. New standard of care for appendiceal epithelial neoplasms and pseudomyxoma peritonei syndrome? Lancet Oncol. 2006;7:69-76.

47. Deraco M, Baratti D, Inglese MG, Allaria B, Andreola S, Gavazzi $\mathrm{C}$, et al. Peritonectomy and intraperitoneal hyperthermic perfusion (IPHP): A strategy that has confirmed its efficacy in patients with pseudomyxoma peritonei. Ann Surg Oncol. 2004;11:393-8.

48. Youssef H, Newman C, Chandrakumaran K, Mohamed F, Cecil TD, Moran BJ. Operative findings, early complications, and long-term survival in 456 patients with pseudomyxoma peritonei syndrome of appendiceal origin. Dis Colon Rectum. 2011;54:293-9.

49. Chua TC, Moran BJ, Sugarbaker PH, Levine EA, Glehen O, Gilly FN, et al. Early- and long-term outcome data of patients with pseudomyxoma peritonei from appendiceal origin treated by a strategy of cytoreductive surgery and hyperthermic intraperitoneal chemotherapy. J Clin Oncol. 2012;30:2449-56.

50. Votanopoulos KI, Shen P, Stewart JH, Levine EA. Current status and future directions in appendiceal cancer with peritoneal dissemination. Surg Oncol Clin N Am. 2012;21:599-609.

51. Gough DB, Donohue JH, Schutt AJ, Gonchoroff N, Goellner JR, Wilson TO, et al. Pseudomyxoma peritonei. Long-term patient survival with an aggressive regional approach. Ann Surg. 1994;219:112-9.

52. Miner TJ, Shia J, Jaques DP, Klimstra DS, Brennan MF, Coit DG. Long-term survival following treatment of pseudomyxoma peritonei: An analysis of surgical therapy. Ann Surg. 2005;241:300-8.

53. Esquivel J, Sugarbaker PH. Second-look surgery in patients with peritoneal dissemination from appendiceal malignancy: Analysis of prognostic factors in 98 patients. Ann Surg. 2001;234:198 205.

54. Sardi A, Jiménez WA, Nieroda C, Sittig M, Macdonald R, Gushchin V. Repeated cytoreductive surgery and hyperthermic intraperitoneal chemotherapy in peritoneal carcinomatosis from appendiceal cancer: Analysis of survival outcomes. Eur J Surg Oncol. 2013;39:1207-13.

55. Votanopoulos KI, Ihemelandu C, Shen P, Stewart JH, Russell GB, Levine EA. Outcomes of repeat cytoreductive surgery with hyperthermic intraperitoneal chemotherapy for the treatment 
of peritoneal surface malignancy. J Am Coll Surg. 2012;215: 412-7.

56. Yan TD, Bijelic L, Sugarbaker PH. Critical analysis of treatment failure after complete cytoreductive surgery and perioperative intraperitoneal chemotherapy for peritoneal dissemination from appendiceal mucinous neoplasms. Ann Surg Oncol. 2007;14:2289-99.

57. Elias D, Honoré C, Ciuchendéa R, Billard V, Raynard B, Lo Dico R, et al. Peritoneal pseudomyxoma: Results of a systematic policy of complete cytoreductive surgery and hyperthermic intraperitoneal chemotherapy. Br J Surg. 2008;95:1164-71.

58. Smeenk RM, Verwaal VJ, Antonini N, Zoetmulder FAN. Survival analysis of pseudomyxoma peritonei patients treated by cytoreductive surgery and hyperthermic intraperitoneal chemotherapy. Ann Surg. 2007;245:104-9.

59. Chua TC, Liauw W, Morris DL. Early recurrence of pseudomyxoma peritonei following treatment failure of cytoreductive surgery and perioperative intraperitoneal chemotherapy is indicative of a poor survival outcome. Int J Colorectal Dis. 2012;27:381-9.

60. Brouquet A, Goéré D, Lefèvre JH, Bonnet S, Dumont F, Raynard $\mathrm{B}$, et al. The second procedure combining complete cytoreductive surgery and intraperitoneal chemotherapy for isolated peritoneal recurrence: Postoperative course and long-term outcome. Ann Surg Oncol. 2009;16:2744-51.

61. Golse N, Bakrin N, Passot G, Mohamed F, Vaudoyer D, Gilly $\mathrm{FN}$, et al. Iterative procedures combining cytoreductive surgery with hyperthermic intraperitoneal chemotherapy for peritoneal recurrence: Postoperative and long-term results. J Surg Oncol. 2012;106:197-203.

Correspondencia: Felipe Solórzano, MD

Correo electrónico: felipesolorzano@gmail.com Bogotá, D.C., Colombia 